\title{
Origin of Endothelium in Human Renal Allografts
}

\author{
R. A. SINCLAIR
}

British Medical fournal, 1972, 4, 15-16

\section{Summary}

Sex chromatin counts performed on the endothelial cells of 40 human kidneys transplanted to recipients of the opposite sex showed that the donor endothelium had persisted except in three poorly functioning and severely damaged grafts. In these a high proportion of the endothelial cells in peritubular capillaries and veins were derived from the host. Endothelial repopulation of organ allografts probably occurs only after severe tissue injury, and it cannot explain the phenomenon of graft adaptation. Repopulated endothelium may be derived from circulating cells.

\section{Introduction}

Woodruff (1959) introduced the concept of transplant "adaptation" to account for the state of relative freedom from rejection which develops in long-term allografts. Medawar (1965) urged investigation of the possibility that adaptation in organ grafts might be explained by an extensive replacement of the donor vascular endothelium by cells derived from the host, and much conflicting evidence has appeared (Alexandre et al., 1963; Stump et al., 1963; O'Neal et al., 1964; Murray et al., 1964; Williams and Alvarez, 1969; Bieber et al., 1970; Bohle and Fischbach, 1970; Williams et al., 1971).

In studying this problem several investigators (Williams and Alvarez, 1969; Bieber et al., 1970; Bohle and Fischbach, 1970; Williams et al., 1971) have used the sex chromatin body as a marker of endothelial origin in transplants performed between the sexes. The usual method has been to examine, "en face," sheets of endothelium prepared by the Hautchen technique. The findings, however, have not been consistent, and in each case endothelium from only the aorta, renal artery, or large renal vessels has been examined, so that the presence or absence of diffuse endothelial repopulation as a basis for graft adaptation can be only inferred. A method has now been developed for counting sex chromatin bodies in the endothelium of vessels of all sizes in sections of paraffin-embedded tissue (Sinclair, 1972). This report presents the results of applying this method to a group of human renal allografts in which a sex difference existed between donor and recipient.

\section{Materials and Method}

The details of tissue processing, staining, and counting techniques have been reported elsewhere (Sinclair, 1972). The material examined came from reserve blocks of formalin-fixed and paraffin-embedded tissue from 45 human renal allografts obtained at open biopsy (16 cases), nephrectomy (10 cases), and necropsy (19 cases). Thionin staining with acid hydrolysis was used throughout. The grafts had survived for between four days and six-and-a-half years, and renal function varied from excellent to very poor. There were 35 male to female grafts, 5 female to male, and 3 male and 2 female sexmatched grafts. A careful check was made from the clinical

\footnotetext{
Department of Pathology, St. Mary's Hospital Medical School, London W.2

R. A. SINCLAIR, M.B., B.S., Research Fellow
}

records of the sex of the donor and the recipient. The donor sex was readily confirmed by an examination of arterial smooth muscle cells, where sexual differentiation is very obvious.

Most of the sections were examined without previous knowledge of the sex of the donor or the recipient. Between 300 and 1,000 endothelial cells were counted from the arteries, peritubular capillaries, vasa recta, and veins from each specimen. Sex chromatin body counts on glomerular endothelium were attempted in only three cases of special interest, for, though they can be seen in normal female glomeruli, nuclear irregularity is very common and confusion with epithelial and mesangial cells is bound to make the counts unreliable. Control values for the frequency of sex chromatin bodies were established in the sexmatched grafts and from earlier results (Sinclair, 1972) in six normal kidneys.

\section{Results}

Mean sex chromatin body control values were $1 \%$ for male and $52 \%$ for female. In 37 of the $\mathbf{4 0}$ cross-sex grafts (Table I) the counts did not differ significantly from normal, thus indicating that the donor endothelium had persisted. Ten of these cases were male to female grafts with sex chromatin body counts of between 3 and $6.5 \%$, but this has not been interpreted as

TABLE I-Sex Chromatin Body (S.C.B.) Counts in 37 Human Renal Allografts showing no Endothelial Repopulation

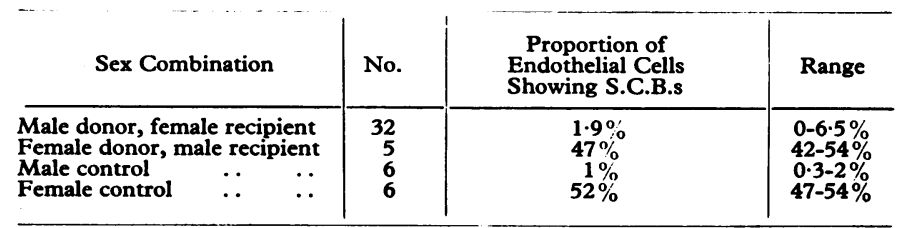

TABLE II-Percentage Sex Chromatin Body Counts in Three Human Renal Allografts showing Endothelial Repopulation

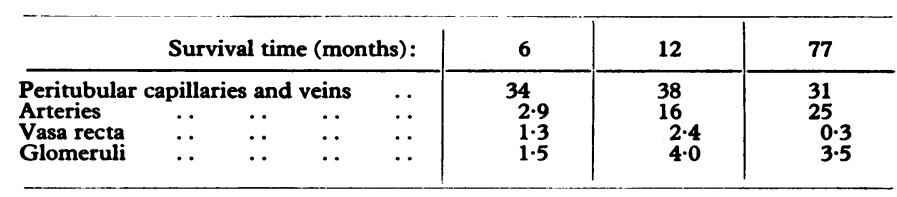

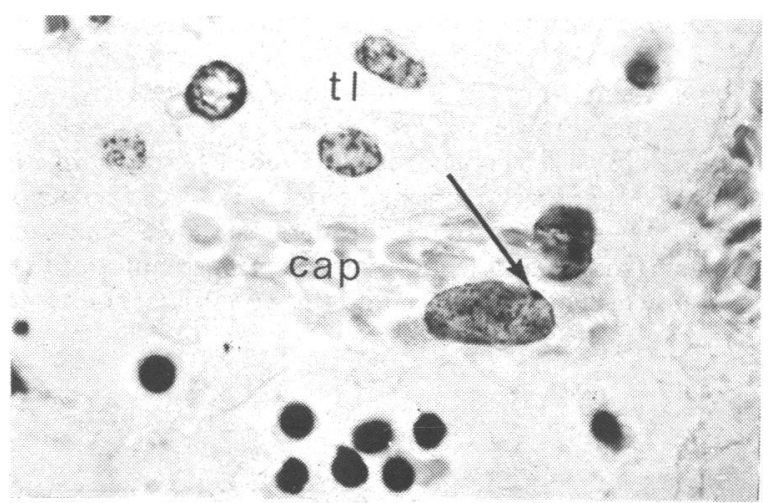

Peritubular capillary from a male to female renal allograft examined at necropsy six months after transplantation. Section includes endothelial nucleus seen full-face which contains well-defined sex chromatin body (arrowed). Tubular nuclei can be seen above, and below are lymphocytes (arrowed). Tubular nuclei can be seen above, and below are lymphocytes
infiltrating interstitium. cap $=$ Peritubular capillary containing red blood infiltrating interstitium. cap $=$ Peritubular capillary containing red blood
cells. $\mathrm{tl}=$ Tubular lumen. (Thionin stain with acid hydrolysis. $\times 1,000$.) 
evidence of a minor degree of endothelial repopulation since occasional confusion of endothelial cells with host-derived fibroblasts (Bohle and Fischbach, 1970) could account for these values. In three grafts, however, endothelial chimerism was clearly present (Table II, see Fig.). These were male kidneys transplanted to female recipients in which the counts in the peritubular capillaries and veins were 38,34 , and $31 \%$. Since only about a half of normal female endothelial cells are seen to contain sex chromatin bodies these values imply that about $60-80 \%$ of the endothelium in these vessels had been replaced by host cells. Counts were also raised in the arteries of two of these grafts, though the values were lower (25 and 16\%) than in the veins and peritubular capillaries. In all three cases the endothelium of the vasa recta had remained donor in origin. Glomerular endothelial counts were attempted in these three kidneys, and in each case only a few sex chromatin bodies were found.

Sixteen of the grafts which showed no evidence of endothelial chimerism were kidneys with good or excellent function and a relatively normal histological picture, each of which had been biopsied electively two years after transplantation. On the other hand, the three kidneys showing evidence of endothelial repopulation possessed minimal function at the time of death or nephrectomy. Histologically two of these three showed areas of recent and old infarction and were more severely damaged than any of the other kidneys in the series. In all three cases there was pronounced vascular narrowing, interstitial fibrosis, and tubular atrophy.

\section{Discussion}

The most important fact to emerge from this study was that although endothelial repopulation can occur it had played no part in protecting the grafted kidneys from immunological attack. On the contrary the evidence suggests that repopulation occurs only after severe tissue injury. Thus none of the wellfunctioning and long-surviving grafts showed repopulation, whereas in the kidneys in which this had occurred renal function was poor and damage due to rejection was extremely severe. These findings may help to resolve the apparent contradictions of previously published work which both discounts (Alexandre et al., 1963; Murray et al., 1964; Bieber et al., 1970; Bohle and Fischbach, 1970) and supports (Stump et al., 1963; O'Neal et al., 1964; Williams and Alvarez, 1969; Williams et al., 1971) the possibility of endothelial repopulation.

Evidence against repopulation has come from the examination of endothelium in the major vessels of cross-sex renal and cardiac allografts (Bieber et al., 1970; Bohle and Fischbach, 1970). In each case the endothelium had remained donor in origin. This does not prove, however, that endothelial repopulation never occurs, since a total of only seven transplants were investigated. In the present series vessels of all sizes were examined from 40 transplants, and endothelial repopulation was present in only three of them. Some other evidence has come from experiments in which well-tolerated renal allografts have survived without immunosuppression when retransplanted to the original donor (Alexandre et al., 1963; Murray et al., 1964). It was argued that foreign antigenicity has.not been incorporated into the kidney during residence in the host, since such a change would have induced rejection after retransplantation. These findings, however, do not preclude the possibility of endothelial repopulation in severely damaged grafts, since in the two cases quoted the renal function was adequate or good before retransplantation and only minor damage was shown histologically.

The formation of a new endothelial layer from host cells $w$ occurs in aortic grafts (Williams and Alvarez, 1969; Williams et al., 1971) and on the surface of Dacron hubs suspended in the aortic blood stream (Stump et al., 1963; O’Neal et al., 1964). The recipients of the aortic grafts did not receive immunosuppressive therapy, and rejection was sufficiently violent to $\stackrel{\vec{S}}{\rightarrow}$ result in several cases of aortic rupture, aneurysm formation, $\bar{O}$ and aortic occlusion. It is to be expected, therefore, that the $\frac{0}{2}$ intima of the transplanted vessels would have been so damaged $\frac{\bar{\omega}}{5}$ that donor-derived cells could not have contributed extensively $\stackrel{\mathbb{Q}}{\propto}$ to the formation of a new endothelial layer. A similar but more extreme situation occurred in the Dacron hub experiments, where the host was the only possible source of endothelium. Disruption of peritubular capillaries (Porter, 1967) and endo- $\overrightarrow{\vec{\omega}}$ thelial loss from larger vessels (Kosek et al., 1969) are features of $\mathscr{\sigma}^{\circ}$ acute rejection in organ allografts. It seems reasonable, there- $\frac{}{3}$ fore, to draw an analogy between the surface of a Dacron hub or a disrupted aortic intima and the vessels of a rejecting renal if allograft. Hence extensive and acute vascular damage would $\underset{\omega}{\infty}$ require repair by host cells while less severely damaged grafts. would be able to restore endothelial continuity from surviving $\vec{v}$ donor elements.

The fact that host repopulation of endothelium occurs at all $v$ raises the question whether the endothelium grows in continuity $\bigcirc$ from the host to the graft or whether the new layer is derived $\frac{f}{\circ}$ from blood-borne cells. Endothelial repopulation was found to $\underset{\mathbb{Q}}{-}$ occur focally in the vascular tree, affecting the peritubular capillaries and veins while sparing the glomerular capillaries, $\vec{\varphi}$ the vasa recta, and the arteries in one case. This distribution $N$ suggests that the new endothelium was derived from circulating cells. This interpretation is consistent with the findings in the Dacron hub experiments and with the evidence that repopulating cells may be derived from the bone marrow (Williams et al., 1971).

This work was supported by a grant from the Simon Marks $\frac{3}{3}$ Charitable Trust Donation and the British Heart Foundation. I wish to thank Professor K. A. Porter for helpful advice, Professor W. S. Peart and Professor T. E. Starzl for providing details of the graft donors and recipients, and Mr. Brian Trefty for expert technical help.

\section{References}

Alexandre, G. P. J., Murray, J. E., Dammin, G. J., and Nolan, B. (1963). 음

Transplantation, 1, 432.
Bieber, C. P., Stinson, E. B., Shumway, N. E., Payne, R., and Kosek, J. O (1970). Circulation, 41, 753 .

Bohle, A., and Fischbach, H. (1970). Virchows Archiv für pathologische $\frac{D}{2}$ Anatomic :nd Physiologie und für klinische Medizin, 349, 170.

Kosek, J. C., Chartrand, C., Hurley, E. J., and Lower, R. R. (1969). Labora- N tory Investigation, $21,328$.

Medawar, P. B. (1965). British Medical Bulletin, 21, 97

Murray, J. E., et al. (1964). Annals of Surgery, 160, 449.

O'Neal,, R. M., Jordan, G. L., Rabin, E. R., DeBakey, M. E., and Halpert, B. (1964). Experimental and Molecular Pathology, 3, 403.

Porter, K. A. (1967). Fournal of Clinical Pathology, 20, Suppl. No. 2, p. 518 .

Sinclair, R. A. (1972). Fournal of Anatomy. In press.

Stump, M. M., Jordan, G. L., DeBakey, M. E., and Halpert, B. (1963) American fournal of Pathology, 43, 361.

Williams, G. M., and Alvarez, C. A. (1969). Surgical Forum, 20, 293.

Williams, G. M., et al. (1971). Transplantation Proceedings, 3, 869.

Woodruff, M. (1959). Biological Problems of Grafting, p. 83 . Oxford, Blackwell Scientific.

\section{.}

